\title{
Article \\ A Large Signal Theory of Multiple Cascaded Bunching Cavities for High-Efficiency Triaxial Klystron Amplifier
}

\author{
Fuxiang Yang, Fangchao Dang *D, Juntao He, Xiaoping Zhang and Jinchuan Ju \\ College of Advanced Interdisciplinary, National University of Defense Technology Changsha, \\ Changsha 410073, China; yangfuxiang@nudt.edu.cn (F.Y.); hejuntao12@163.com (J.H.); \\ zhangxiaoping@nudt.edu.cn (X.Z.); jujinchuan@126.com (J.J.) \\ * Correspondence: dangfangchao_nudt@163.com
}

check for updates

Citation: Yang, F.; Dang, F.; He, J.; Zhang, X.; Ju, J. A Large Signal Theory of Multiple Cascaded Bunching Cavities for High-Efficiency Triaxial Klystron Amplifier. Electronics 2021, 10, 1284. https:// doi.org/10.3390/electronics10111284

Academic Editor: Rocco Giofre

Received: 22 April 2021

Accepted: 25 May 2021

Published: 28 May 2021

Publisher's Note: MDPI stays neutral with regard to jurisdictional claims in published maps and institutional affiliations.

Copyright: (c) 2021 by the authors. Licensee MDPI, Basel, Switzerland. This article is an open access article distributed under the terms and conditions of the Creative Commons Attribution (CC BY) license (https:// creativecommons.org/licenses/by/ $4.0 /)$.

\begin{abstract}
This paper presents a large signal theory of multiple cascaded bunching cavities for the design of high-efficiency triaxial klystron amplifiers (TKAs). The theoretical analysis of multiple cascaded bunching cavities is presented, focusing on the relationship between gap voltage and first harmonic current and velocity dispersion, which can exactly describe the clustering state of intense relativistic electron beams. The theoretical results of the first harmonic current and velocity dispersion are basically consistent with its simulation results, which can justify a high degree of confidence in the validity of that theory. This theory can predict the possibility of deep modulation of intense relativistic electron beams when the depth of the first harmonic current is about $150 \%$ by multiple cascaded bunching cavities. By properly accounting for this theory, we can design a Ku-band TKA with nearly 60\% microwave conversion efficiency, which can provide theoretical and simulation guidance for the design of high-efficiency TKAs. More importantly, when we increase the electron beam voltage from $300 \mathrm{kV}$ to $600 \mathrm{kV}$ and keep the relativistic perveance constant, this device also can obtain more than $50 \%$ efficiency and $40 \mathrm{~dB}$ gain. As a result, we can design a Ku-band TKA with high average output power of about $1.5 \mathrm{GW}, 52 \%$ efficiency and $46 \mathrm{~dB}$ gain.
\end{abstract}

Keywords: large signal theory; high-efficiency; triaxial klystron amplifier

\section{Introduction}

Coherent power combine is one of the most important directions in the high-power microwave (HPM) field, while the research of 100 gigawatts HPM sources is gradually an important trend [1-4]. Relativistic klystron amplifiers (RKAs) have been considered as an effective method to realize coherent power combine, which can achieve an equivalent radiant power beyond the breakdown limit. So far, L-band, S-band and X-band RKAs have been obtained by GW-level output power and hundred nanosecond output [5-8]. As the frequency of HPM sources rises to the Ku-band, the power handling capability decreases with small size of devices [9]. As a result, problems like RF breakdown and pulse shortening would be produced, which greatly hinders the development of high-power and long-pulse HPM sources.

Triaxial klystron amplifiers (TKAs) with a large radius have power handling capability, which can be a potential Ku-band HPM source to obtain GW-level output power. However, the efficiency of TKAs is still less than $40 \%$, while traditional KW-level klystrons can obtain a more than $80 \%$ RF power production efficiency [10,11]. The design of traditional KW-level klystrons proposes several methods, such as multiple cascaded bunching cavities, long drift tube length, and a second harmonic cavity, to bunch electrons as soon as possible and achieve high-efficiency as a result. According to the design of traditional KW-level klystrons, it is clear that deep modulation of the electron beam is essential for a high microwave conversion efficiency. Considering the particularity of TKAs, we choose multiple cascaded bunching cavities to interact with the electron beams to obtain deeply clustered electrons. 
The design and optimization of a large radius Ku-band TKA, with multiple cascaded bunching cavities, is time-consuming if we just depend on particle-in-cell simulation. The modulation theory of multiple cascaded bunching cavities is an effective method to shorten the optimization process. The modulation model of a single cavity has been analyzed before [12,13], and we need to improve the modulation model of multiple cascaded bunching cavities to obtain deeply clustering electrons. In the modulation model of the multiple cascaded bunching cavities, the gap voltage of the bunching cavity is close to the electron beam voltage so that the small signal theory loses its effect. Therefore, we need develop a large signal theory of multiple cascaded bunching cavities to guide the design of high-efficiency Ku-band TKAs.

This paper comprises four parts as follow. In Section 2, the theoretical analysis of the multiple cascaded bunching cavities' modulation in TKAs is derived. In Section 3, the numerical results of the multiple cascaded bunching cavities are given and compared with the simulation results at the same time. Then, a high-efficiency and high-power Ku-band TKA is designed in Section 4. Finally, we draw a brief conclusion in Section 5.

\section{Model Description}

Figure 1 illustrates the schematic of a multiple cascaded cavities Ku-band TKA, which contains a cathode, input cavity, second bunching cavity, third bunching cavity and output cavity. Electrons emit from the cathode, then modulate sequentially by the input cavity, second bunching cavity, third bunching cavity, and eventually extract power at the output cavity. The input cavity introduces weak velocity modulation to electrons by an external RF signal, and the weak velocity modulation is changed into density modulation when they pass gradually through the drift tube between the input and second bunching cavity. Then, weak density modulation strengthens gradually in the second and third bunching cavity. At last, the deeply modulated electron beam interacts with the output cavity and loses energy to the microwaves. In our model, we analyze the modulation of the input cavity, second and third bunching cavity, respectively, in theory, which aims to conclude the relationship between the gap voltage of each cavity and first harmonic current and velocity dispersion. According to the theoretical analysis, we can obtain the optimal gap voltage of each bunching cavity, which can produce a deeply clustering condition of electrons.

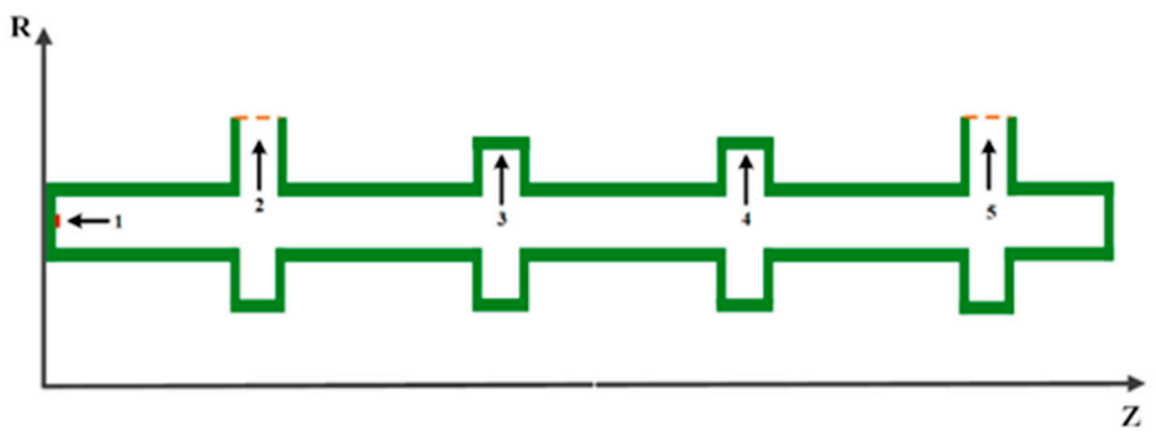

Figure 1. The schematic of a multiple cascaded cavities TKA: 1—cathode, 2-input cavity, 3-second bunching cavity, 4-third bunching cavity, 5-output cavity.

To simplify the problem, we assume electrons only move along the axial direction, that is to say, we assume electrons have only axial velocity under a large magnetic field and there is no radial velocity. Following the electron beam "debunching" theory [12-14], the displacement of the modulated electron beam can be expressed as follows:

$$
\frac{d^{2}}{d t^{2}} z=-\omega_{p}^{2} z
$$

where $z$ is the displacement of modulated electron beam, and $\omega_{p}$ is plasma frequency associated with intense electron beam. Therefore, considering space charge effects and 
relativistic effect, the initial velocity modulation caused by an external rf signal in input cavity can be described as follows:

$$
v_{1}=\varepsilon_{1} v_{0} \sin \left(\omega t_{1}\right) \cos \beta_{p} z
$$

where $v_{0}$ is initial velocity, $\omega$ is working frequency of the input cavity, $t_{1}$ is $\beta_{p}=\omega_{p} / v_{0}$, and $\varepsilon_{1}$ is modulation coefficient, which can be approximated as follows:

$$
\varepsilon_{1}=\left|\sqrt{1-\left(1+\frac{V_{e}+V_{1}}{511}\right)^{-2}} / \sqrt{1-\left(1+\frac{V_{e}}{511}\right)^{-2}}-1\right|
$$

where $V_{e}$ and $V_{1}$ are electron beam voltage and modulation voltage of input cavity, respectively. Then, we can conclude the phase relation between $t=t_{1}$ and $t=t_{2}$ as follows:

$$
\omega t_{2}=\omega t_{1}+\theta_{12}-X_{12} \sin \omega t_{1}
$$

where $\theta_{12}=\omega l_{12} / v_{0}, X_{12}=\varepsilon_{1} \theta_{12} \frac{\sin \beta_{p} l_{12}}{\beta_{p} l_{12}}, l_{12}$ is the length between cathode and second bunching cavity. The first harmonic frequency current is given by the following:

$$
I_{1}=2 I_{0} J_{n}(n \bar{V} \sin \bar{z})
$$

where $\bar{V}=\frac{1}{2}\left(\varepsilon_{1} \omega / \omega_{p}\right), \bar{z}=\omega_{p} z / v_{0}$.

According to the analysis above, we assume the total velocity modulation is the sum of the initial velocity and second velocity modulation caused by the cathode and second bunching cavity, respectively [12].

$$
v_{2}=\varepsilon_{1} v_{0} \sin \left(\omega t_{1}\right) \cos \beta_{p} z+\varepsilon_{2} v_{0} \sin \left(\omega t_{1}-X_{12} \sin \omega t_{1}\right) \cos \beta_{p}\left(z-l_{12}\right)
$$

where $\varepsilon_{2}=\left|\sqrt{1-\left(1+\frac{V_{e}+V_{2}}{511}\right)^{-2}} / \sqrt{1-\left(1+\frac{V_{e}}{511}\right)^{-2}}-1\right|, V_{2}$ are the electron beam voltage and modulation voltage of preliminary bunching. Then, we can conclude phase relation between $t=t_{1}$ and $t=t_{3}$ as follows:

$$
v_{2}=\varepsilon_{1} v_{0} \sin \left(\omega t_{1}\right) \cos \beta_{p} z+\varepsilon_{2} v_{0} \sin \left(\omega t_{1}-X_{12} \sin \omega t_{1}\right) \cos \beta_{p}\left(z-l_{12}\right)
$$

where $\theta_{13}=\omega l_{13} / v_{0}, X_{13}=\varepsilon_{1} \theta_{13} \frac{\sin \beta_{p} l_{13}}{\beta_{p} l_{13}}, \theta_{23}=\omega l_{23} / v_{0}, X_{23}=\varepsilon_{2} \theta_{23} \frac{\sin \beta_{p} l_{23}}{\beta_{p} l_{23}}, l_{13}$ is the length between the cathode and second bunching cavity, while $l_{23}$ is the length between the second and third bunching cavity. Sine function can be represented by Bessel function by using the Fourier series as in [12].

$$
\begin{aligned}
\sin \left(\omega t_{1}-X_{12} \sin \omega t_{1}\right) & =\sum_{n=1}^{\infty}(-1)^{n-1} J_{n-1}\left(X_{12}\right) \sin \left(n \omega t_{1}\right)-\sum_{n=0}^{\infty} J_{n+1}\left(X_{12}\right) \sin \left(n \omega t_{1}\right) \\
I_{1} & =2 I_{0} \sum_{p=1}^{\infty} \sum_{n=-\infty}^{\infty} J_{p}\left(X_{13}\right) J_{n}\left(X_{23}\right) J_{n+p-1}\left(n X_{12}\right)
\end{aligned}
$$

As follows, total velocity modulation can be assumed as the sum of the initial velocity and second velocity modulation caused by the cathode with a preliminary bunching signal, second and third bunching cavity, respectively.

$$
v_{2}=\varepsilon_{1} v_{0} \sin \left(\omega t_{1}\right) \cos \beta_{p} z+\varepsilon_{2} v_{0} \sin \left(\omega t_{1}-X_{12} \sin \omega t_{1}\right) \cos \beta_{p}\left(z-l_{12}\right)
$$

where $\varepsilon_{3}=\left|\sqrt{1-\left(1+\frac{V_{e}+V_{3}}{511}\right)^{-2}} / \sqrt{1-\left(1+\frac{V_{e}}{511}\right)^{-2}}-1\right|, V_{3}$ are electron beam voltage and modulation voltage of second bunching cavity. 
In the multiple cavities TKA, the preliminary modulation theory of the electron beam is much smaller than the second and third bunching cavity; therefore, we assume the velocity with the input cavity's modulation is still close to $v_{0}$, that is to say, the modulation process of the second and third bunching cavity is approximately equal to the first and second bunching cavity discussed before. Certainly, this assumption is not completely accurate because it ignores any speed change in the input cavity. The theoretical value may be slightly smaller than the actual value.

\section{Model Verification and Optimization}

In this part, we verify the accuracy of the deep modulation theory by comparing theoretical results with the simulation results. Figure 2 shows the modulation model of the input cavity, it is clear that the theoretical results of first harmonic current with different modulation voltages coincide well with the simulation results given by PIC code KARAT in Figure 3a [15]. As Figure 3a shows, the modulation voltage rises from $50 \mathrm{kV}$ to $200 \mathrm{kV}$, electron clustering gradually accelerates, and the maximum value of first harmonic current increases. The maximum depth of the first harmonic current reaches about $120 \%$ when the modulation voltage is $200 \mathrm{kV}$ in the input cavity. If we continue to increase the modulation voltage to $300 \mathrm{kV}$, there is strong electron overtaking so that the maximum depth of the first harmonic current decreases to only $100 \%$.

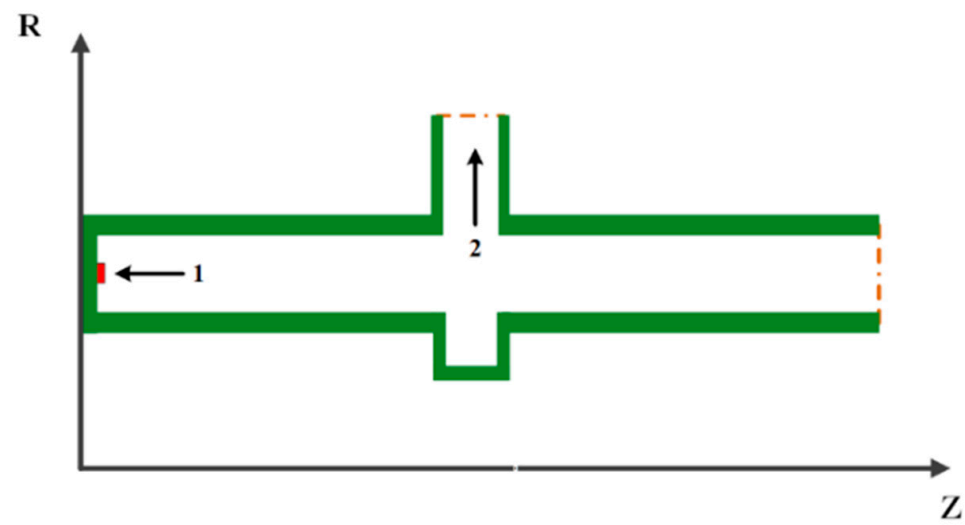

Figure 2. The modulation model of input cavity: 1 -cathode, 2 -input cavity.

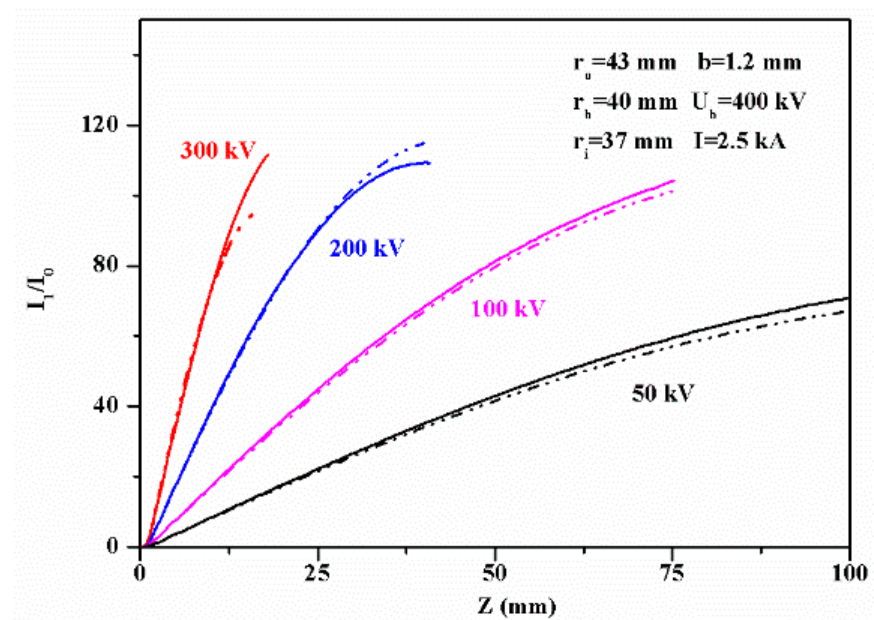

(a)

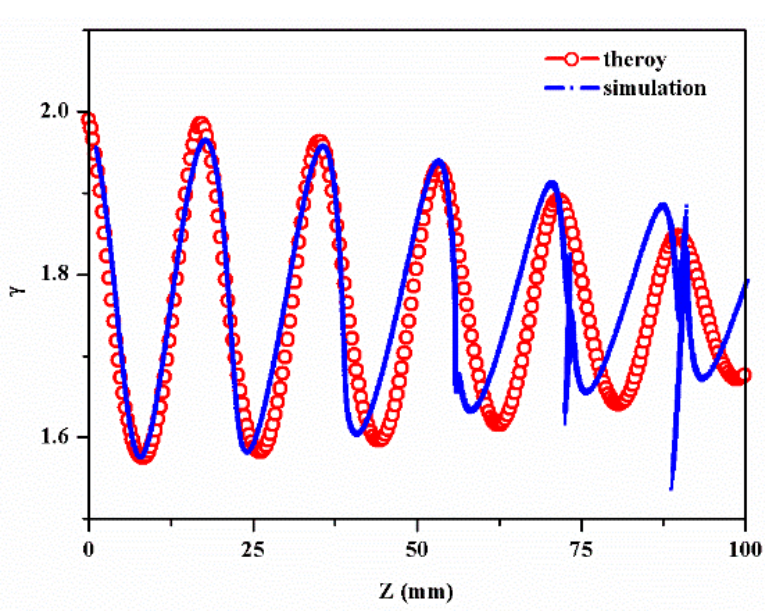

(b)

Figure 3. (a) The first harmonic current versus different modulation voltages: full lines show theoretical results while dotted lines show simulation results. (b) The velocity dispersion versus displacement $Z$. 
The theoretical efficiency of TKA is related to not only the first harmonic current, but also velocity dispersion, which can be defined as follows:

$$
\eta=\frac{1}{2}\left|\frac{I_{1}}{I_{0}}\right|\left(\frac{\gamma_{\min }-1}{\gamma_{0}-1}\right)^{1 / 2}
$$

Therefore, it is necessary to calculate velocity dispersion after the input cavity's modulation. Figure $3 b$ illustrates velocity dispersion with the input cavity's modulation versus displacement. When modulated electrons drift about $50 \mathrm{~mm}$ in the drift tube, the electron-clustering state is best. Then, the transcendence phenomenon occurs when the drift tube length increases. This theory cannot describe the state of the electrons after the transcendence phenomenon occurs.

Figure 4 shows the modulation model of the input and second bunching cavity. The theoretical results also show good consistency with the simulation results in Figure 5a,b. That means that the deduced first harmonic current equations have high accuracy and are valid to estimate a real first harmonic current. When the modulation voltage is $80 \mathrm{kV}$ in the input cavity, the maximum depth of the first harmonic current reaches about $140 \%$, which is larger than that in the modulation of the input cavity. That means that two bunching cavities can increase the depth of the first harmonic current to obtain deep modulation electrons. The velocity dispersion of the first and second bunching cavity's modulation, showed in Figure 5b, is larger than it is of the input cavity's modulation in Figure 3b, which indicates modulation of multiple cavities can increase the velocity dispersion of electrons. We need to consider both factors at the same time in the design of high-efficiency RKAs.

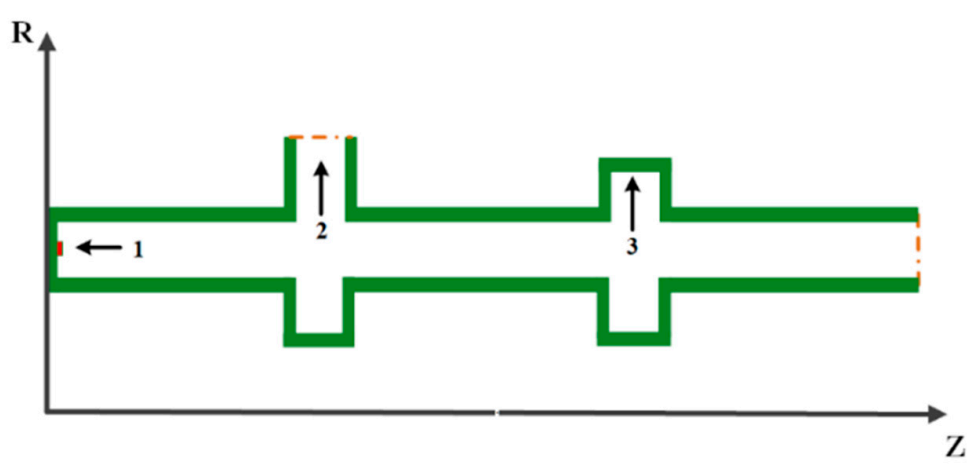

Figure 4. The modulation model of input and second bunching cavity: 1-cathode, 2-input cavity, 3-second bunching cavity.

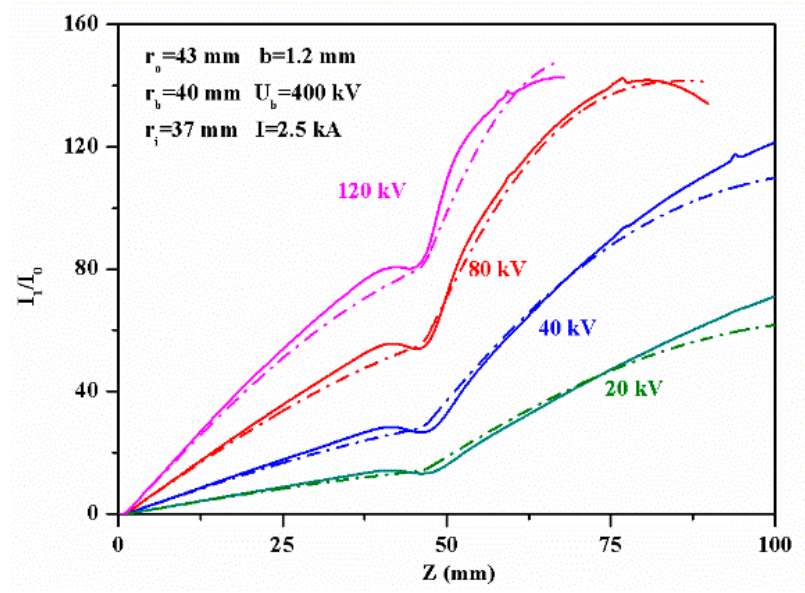

(a)

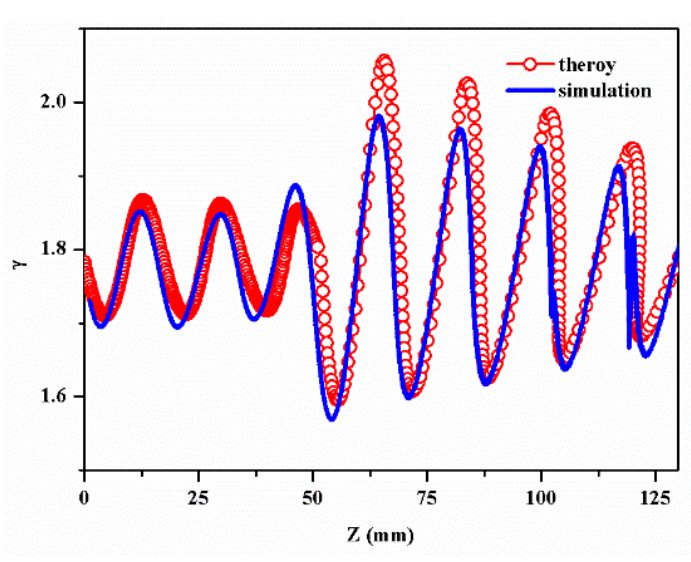

(b)

Figure 5. (a) The first harmonic current versus different modulation voltage: full lines show theoretical results while dotted lines show simulation results. (b) The velocity dispersion versus displacement $Z$. 
Figure 6 illustrates the modulation model of the first, second and third bunching cavities. When the modulation voltage is $20 \mathrm{kV}$ in the input cavity, the maximum depth of the first harmonic current reaches more than $160 \%$ in Figure $7 \mathrm{a}$, which is larger than that in the modulation of the first and second bunching cavities. That means three bunching cavities can rise the maximum depth of the bunching electrons.

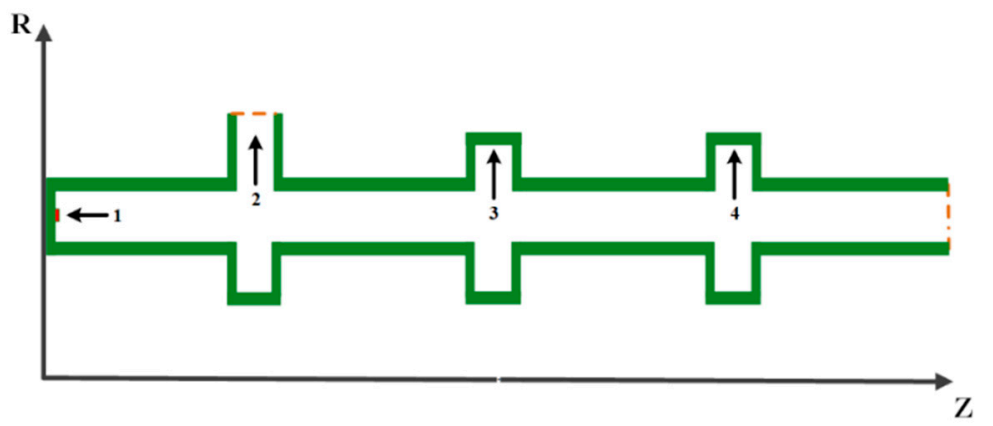

Figure 6. The modulation model of first, second and third bunching cavity: 1—cathode, 2 -input cavity, 3-second bunching cavity.

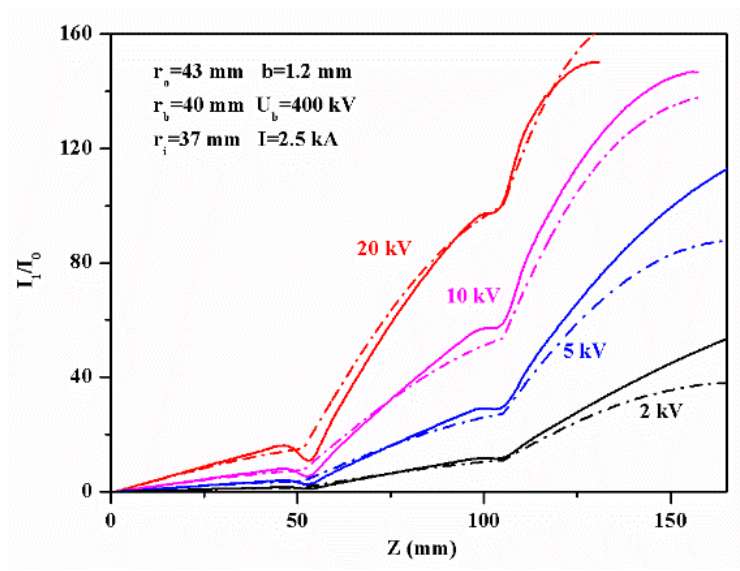

(a)

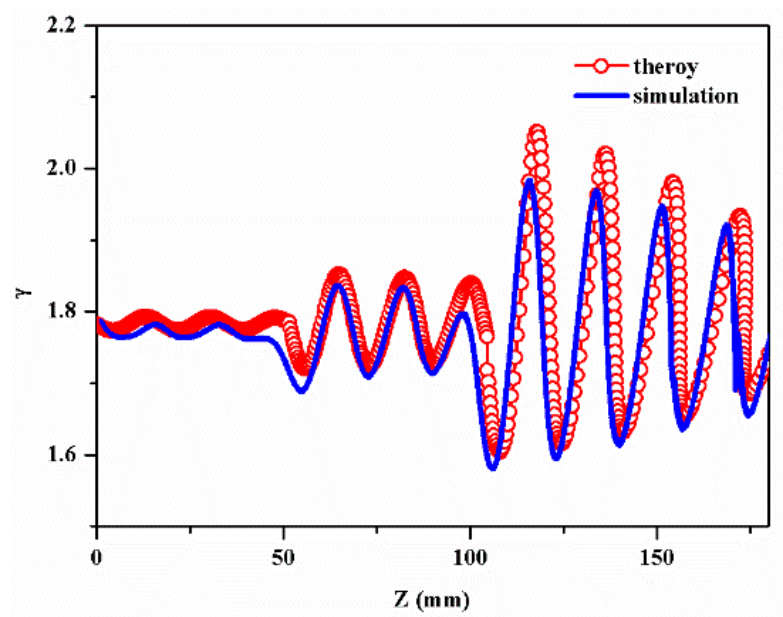

(b)

Figure 7. (a) The first harmonic current versus different modulation voltage: full lines show theoretical results while dotted lines show simulation results. (b) The velocity dispersion versus displacement $Z$.

We compare the theoretical results of velocity dispersion with the simulation results when the modulation voltage is $10 \mathrm{kV}$, which also shows good consistency in Figure $7 \mathrm{~b}$. The velocity dispersion of the first, second and third bunching cavity's modulation showed in Figure $7 \mathrm{~b}$ is similar to the first and second bunching cavity's modulation in Figure $5 \mathrm{~b}$. As Figure 7a shows, when the modulation voltage is only $2 \mathrm{kV}$ and $5 \mathrm{kV}$, the theoretical results of the first harmonic current are less than the simulation results. This is consistent with the analysis of the assumption, and this error needs to be corrected in future work.

\section{Design for a High-Efficiency TKA}

With the analysis of the multiple bunching cavity's modulation, we can design a five-cavities Ku-band TKA with a first, second and third bunching cavity. The klystron parameters are summarized in Table 1. 
Table 1. Parameters of the multiple cavities RKA.

\begin{tabular}{ccccc}
\hline & Input Cavity & $\begin{array}{c}\text { 2nd Bunching } \\
\text { Cavity }\end{array}$ & $\begin{array}{c}\text { 3rd Bunching } \\
\text { Cavity }\end{array}$ & Output Cavity \\
\hline $\mathrm{f}(\mathrm{GHz})$ & 14.25 & 14.26 & 14.34 & 14.25 \\
$\mathrm{Q}$ & 354 & 745 & 312 & 50 \\
$\mathrm{M}$ & 0.484 & 0.638 & 0.605 & 0.743 \\
$\mathrm{R} / \mathrm{Q}(\Omega)$ & 3.6 & 3.1 & 4.2 & 2.5 \\
\hline
\end{tabular}

This device obtains a deep modulation intense electron beam with nearly $150 \%$ first harmonic current, as shown in Figure 8. Therefore, we can design a TKA with nearly $60 \%$ efficiency when the electron beam voltage is $400 \mathrm{kV}$ and the beam current is $2.5 \mathrm{kA}$, as in Figure 9a,b. The output power is stable and the frequency spectrum is pure, which indicates that there is no pulse shortening and self-oscillation in our device.

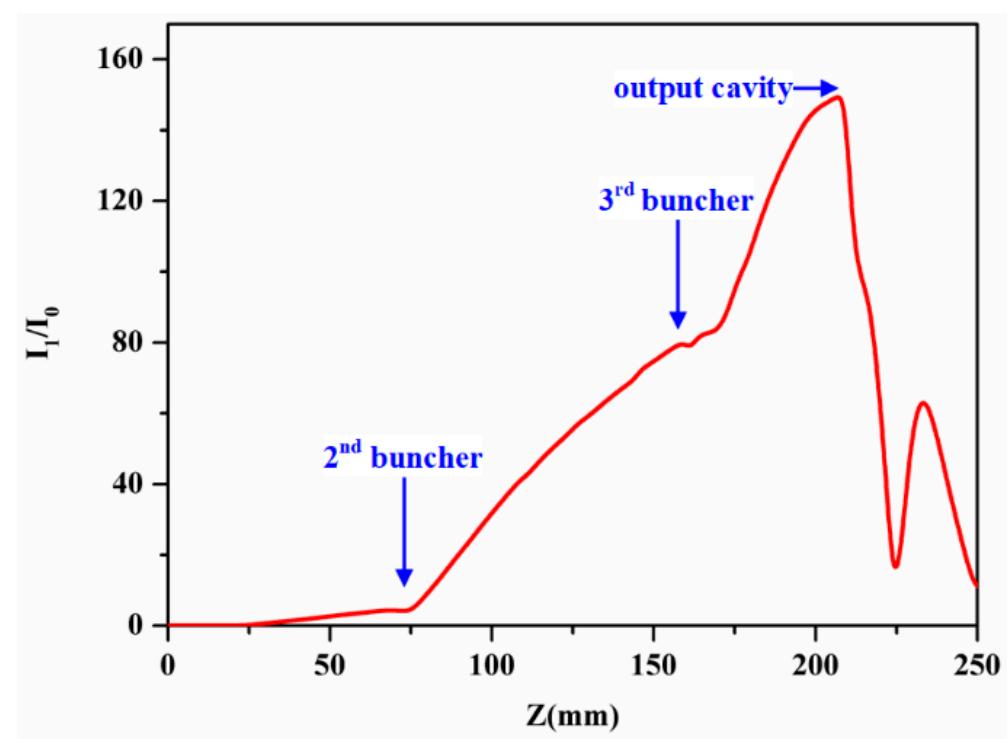

Figure 8. The first harmonic current with the deep modulation of a preliminary bunching signal, second and third bunching cavity.

a)

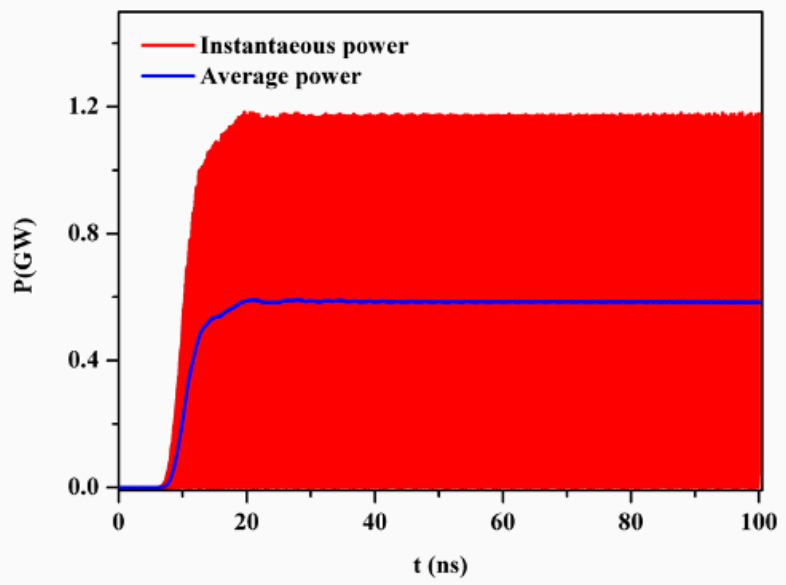

b)

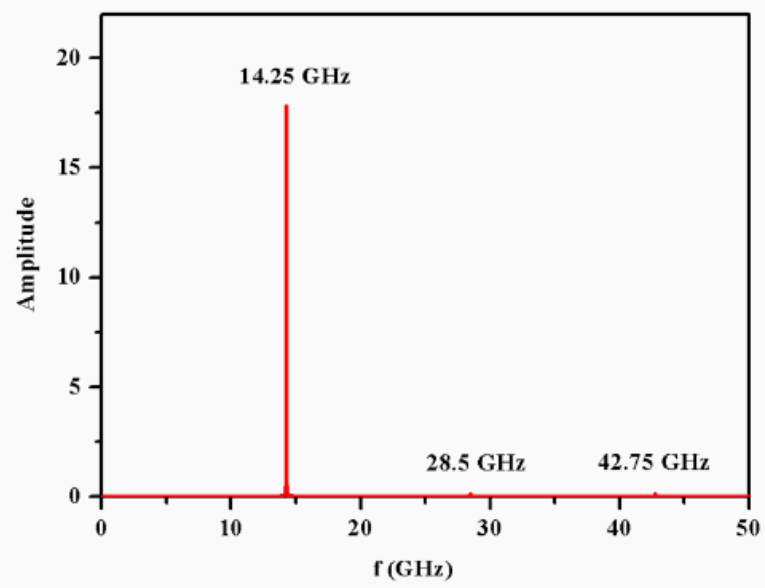

Figure 9. (a) Output microwave power versus time. (b) Frequency spectrum of output signal.

Additionally, when the electron beam voltage varies from $300 \mathrm{kV}$ to $600 \mathrm{kV}$ and the relativistic perveance stays constant, the first harmonic current has the same trend shown in 
Figure 10. It means that the device can operate with the electron beam, no matter how the electron beam voltage changes, as long as the perveance remains unchanged. This device is insensitive to voltage, which is different from other high-power microwave generators [16]. The maximum depth of the first harmonic current reaches about $145 \%$ when the electron beam voltage and current are $600 \mathrm{kV}$ and $4.8 \mathrm{kA}$, respectively.

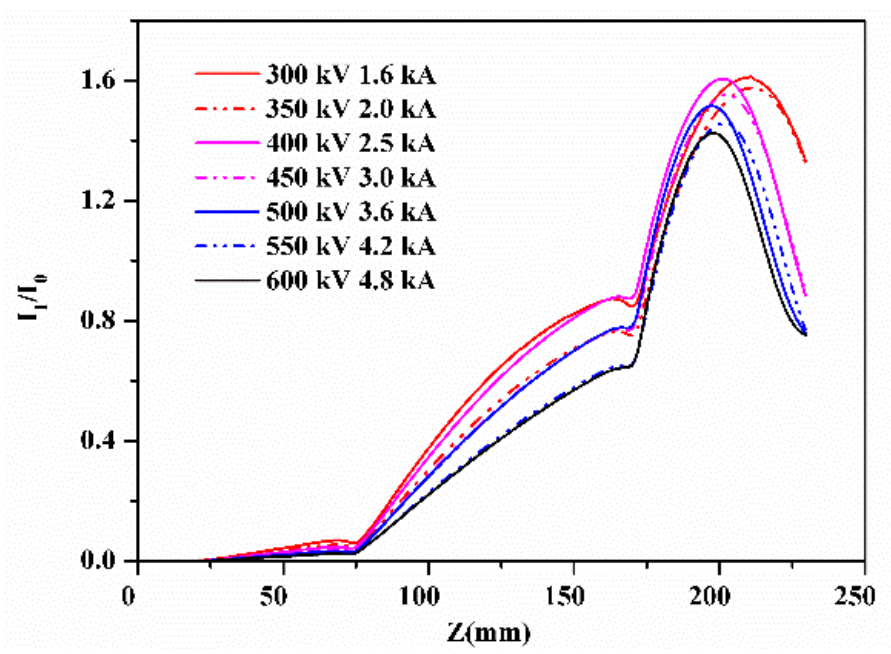

Figure 10. The first harmonic current distribution versus electron beam voltage and current.

Besides, as Figure 11 shows, the gain of device increases as the electron beam voltage increases. This device can obtain more than $50 \%$ efficiency and $40 \mathrm{~dB}$ gain. At a result, the TKA can achieve about $1.5 \mathrm{GW}$ high output power at $14.25 \mathrm{GHz}$, with nearly $52 \%$ efficiency and $46 \mathrm{~dB}$ gain. Figure 12a,b show the phase-locking characteristics of that device. When the input frequency increases from $14.22 \mathrm{GHz}$ to14.28 GHz, or the beam voltage rises from $300 \mathrm{kV}$ to $500 \mathrm{kV}$, the output microwave phase can be locked well. This device has a steady working condition.

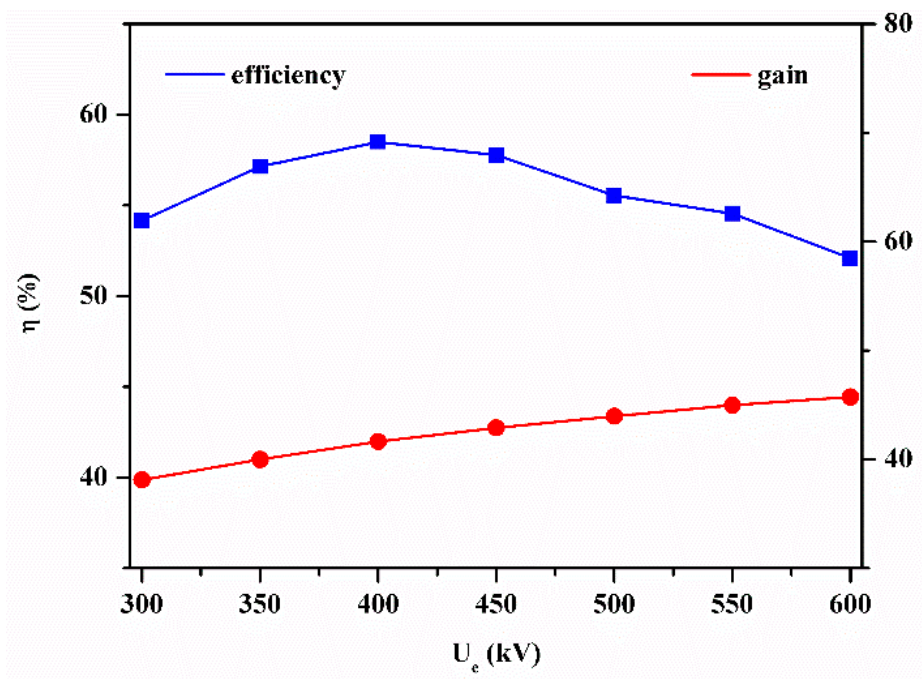

Figure 11. The efficiency and gain versus electron beam voltage. 

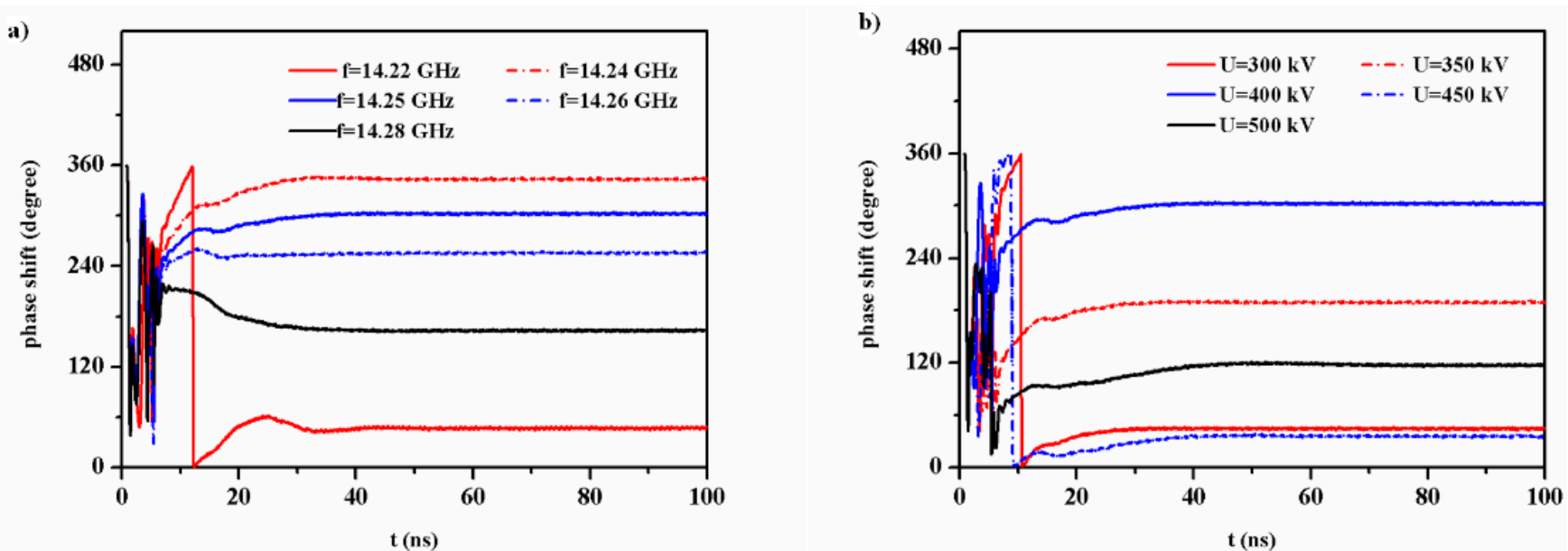

Figure 12. (a) Phase shift versus input frequency. (b) Phase shift versus diode voltages.

\section{Conclusions}

This paper describes a large signal theory for the design of high-efficiency TKA. With the electron kinematics equation and "debunching" theory, we analyzed the modulation of first, second and third bunching cavities, respectively. The comparisons of the theoretical and simulation results show excellent agreement and justify a high degree of confidence in the validity of this theory. By using multiple cascaded bunching cavities, intense relativistic electron beams can cluster very closely, and the depth of the first harmonic current is nearly $150 \%$. With the guidance of the deep modulation theory, we can obtain the optimal gap voltage of each bunching cavity and design a TKA with about $60 \%$ output efficiency as a result. Additionally, this device can operate stably when the electron beam voltage increases from $300 \mathrm{kV}$ to $600 \mathrm{kV}$, and we obtain a TKA with an average output power of about $1.5 \mathrm{GW}$ at $14.25 \mathrm{GHz}$, with nearly $52 \%$ efficiency and $46 \mathrm{~dB}$ gain.

Author Contributions: Conceptualization, F.D. and J.H.; methodology, F.D. and J.H.; supervision, F.D., J.H., X.Z. and J.J.; validation, F.Y. and F.D.; writing-original draft, F.Y.; writing-review and editing, F.Y. and F.D. All authors have read and agreed to the published version of the manuscript.

Funding: This research received no external funding.

Data Availability Statement: The data presented in this study are available on request from the corresponding author.

Conflicts of Interest: The authors declare no conflict of interest.

\section{References}

1. Schamiloglu, E. High power microwave sources and applications. In Proceedings of the International Microwave Symposium Digest, Fort Worth, TX, USA, 6-11 June 2004.

2. Benford, J. High Power Microwaves, 2nd ed.; John, S., Edl, S., James, B., Eds.; CRC Press-Taylor \& Francis: London, UK, 2007; Volume 3, pp. 43-97.

3. Zhang, J.; Zhang, W.; Zhang, D.; Ju, J.; Zhong, H. Suppression of the Higher-Order Azimuthal Mode Competition in an X-Band Triaxial Klystron Amplifier with a Slotted Coaxial Waveguide. IEEE Trans. Electron Device 2021, 67, 1215-1220. [CrossRef]

4. Zhang, J.; Zhang, D.; Fan, Y.; He, J.; Xun, T. Progress in narrowband high-power microwave sources. Phys. Plasmas 2020, 27, 010501. [CrossRef]

5. Ju, J.; Zhang, J.; Qi, Z.; Yang, J.; Shu, T.; Zhang, J.; Zhong, H. Towards coherent combining of X-band high power microwaves: Phase-locked long pulse radiations by a relativistic triaxial klystron amplifier. Sci. Rep. 2016, 6, 30657. [CrossRef] [PubMed]

6. Liu, Z.; Huang, H.; Jin, X.; Li, S.; Wang, T.; Fang, X. Investigation of an X-Band Long Pulse High-Power High-Gain Coaxial Multibeam Relativistic Klystron Amplifier. IEEE Trans. Electron Devices 2018, 66, 722-728. [CrossRef]

7. Liu, Z.; Huang, H.; Jin, X.; Li, S.; Wang, T.; Fang, X. Investigation of the phase stability of an X-band long pulse multibeam relativistic klystron amplifier. Phys. Plasmas 2016, 23, 093110. [CrossRef]

8. Zhang, W.; Ju, J.; Zhang, J.; Zhong, H. A high-gain and high-efficiency X-band triaxial klystron amplifier with two-stage cascaded bunching cavities. Phys. Plasmas 2017, 24, 123118. [CrossRef] 
9. Dang, F.; Zhang, X.; Zhong, H.; Zhang, J.; Ju, J. A high efficiency Ku-band radial line relativistic klystron amplifier. Phys. Plasmas 2016, 23, 073113.

10. Baikov, A.Y.; Marrelli, C.; Syratchev, I. Toward High-Power Klystrons with RF Power Conversion Efficiency on the Order of $90 \%$. IEEE Trans. Electron Devices 2015, 62, 3406-3412. [CrossRef]

11. Cai, J.; Syratchev, I.; Lui, Z.; Liu, Z. Scaling Procedures and Post-Optimization for the Design of High-Efficiency Klystrons. IEEE Trans. Electron Devices 2019, 66, 1075-1081. [CrossRef]

12. Qi, Z.; Zhang, J.; Zhong, H.; Zhang, Z. A large-signal theory of bunching in the triaxial klystron amplifier. Laser Part. Beams 2012, 30, 569-574. [CrossRef]

13. Friedman, M.; Krall, J.; Lau, Y.Y.; Serlin, V. Externally modulated intense relativistic electron beams. J. Appl. Phys. 1988, 64, 3353-3379. [CrossRef]

14. Xie, J.; Zhao, Y. Klystron Cluster Theory; Science Press: Beijing, China, 1966.

15. Tarakanov, V.P. User's Manual for Code KARAT, Sodas Software Version Fund Belgique; Berkeley Research Associates: Emeryville, CA, USA, 1992.

16. Yang, F.; Zhang, X. A Frequency-Tunable V-Band Radial Relativistic Backward-Wave Oscillator. IEEE Trans. Plasma Sci. 2019, 47, 2562-2566. [CrossRef] 